\title{
Comparação do desfecho radiográfico das escolioses idiopáticas do adolescente tratadas com instrumentação híbrida, parafusos pediculares ou ganchos
}

Comparison of the radiographic outcomes using hybrid constructs, pedicle screws or hook instrumentation for the treatment of idiopathic scoliosis

Comparación de los resultados radiológicos en el tratamiento de la escoliosis idiopática del adolescente utilizando instrumentación híbrida, tornillos pediculares o ganchos

\author{
Denis Seguchi Sakai ${ }^{1}$ \\ Ricardo Shigueaki Galhego Umeta ${ }^{2}$ \\ Maria Fernanda Silber Caffaro ${ }^{3}$ \\ Robert Meves ${ }^{4}$ \\ Elcio Landim 5 \\ Osmar Avanzi ${ }^{6}$
}

\section{RESUMO}

Objetivos: comparar os resultados radiográficos dos pacientes portadores de escoliose idiopática do adolescente (EIA) tratados cirurgicamente, com instrumentação com ganchos, híbrida ou parafusos pediculares, com seguimento mínimo de um ano. Métodos: avaliação radiográfica retrospectiva dos pacientes portadores de escoliose idiopática do adolescente tratados com diferentes instrumentações, nos períodos pré-operatório, pós-operatório imediato e pós-operatório de um ano utilizando o método de medição de Cobb. Resultados: não houve dife-

\section{ABSTRACT}

Objectives: to compare the radiographic outcomes of patients with adolescent idiopathic scoliosis (AIS) treated surgically with three different instrumentations (hooks only, hybrid or pedicular screws), with minimal of one-year follow-up. Methods: retrospective radiographic evaluation of patients with idiopathic scoliosis treated with different instrumentations, in the preoperative, postoperative and one year postoperative follow-up using the Cobb method. Results: no differences were found in the behavior

\section{RESUMEN}

Objetivos: comparar los hallazgos radiográficos de los pacientes con escoliosis idiopática del adolescente (EIA) tratados quirúrgicamente, con una instrumentación con ganchos, tornillos pediculares o híbridos, y un seguimiento mínimo del uno año. Métodos: evaluación retrospectivo de las radiografías de los pacientes con escoliosis idiopática tratados con diferentes instrumentaciones, en el pre y postoperatorio y un año después de la operación utilizando el método de medición de Cobb. Resultados: no hubo diferencias en el compor-

\footnotetext{
Trabalho realizado no Departamento de Ortopedia e Traumatologia da Faculdade de Ciências Médicas da Santa Casa de Misericórdia de São Paulo - FCMSCSP São Paulo (SP), Brasil.

'Aluno do Curso de Aperfeiçoamento do Grupo de Coluna do Departamento de Ortopedia e Traumatologia da Faculdade de Ciências Médicas da Santa Casa de Misericórdia de São Paulo - FCMSCSP - São Paulo (SP), Brasil.

${ }^{2}$ Médico; Pós-graduando do Grupo de Coluna do Departamento de Ortopedia e Traumatologia da Faculdade de Ciências Médicas da Santa Casa de Misericórdia de São Paulo - FCMSCSP - São Paulo (SP), Brasil.

${ }_{3}^{3}$ Professora Instrutora e Médica Assistente do Grupo de Coluna do Departamento de Ortopedia e Traumatologia da Faculdade de Ciências Médicas da Santa Casa de Misericórdia de São Paulo - FCMSCSP - São Paulo (SP), Brasil.

${ }^{4}$ Chefe do Grupo da Coluna Doutor; Professor do Departamento de Ortopedia e Traumatologia da Santa Casa de Misericórdia de São Paulo - SCMSP - São Paulo (SP), Brasil.

${ }^{5}$ Doutor; Professor e Médico Consultor do Grupo de Coluna do Departamento de Ortopedia e Traumatologia da Faculdade de Ciências Médicas da Santa Casa de Misericórdia de São Paulo - FCMSCSP - São Paulo (SP), Brasil.

${ }^{6}$ Doutor; Professor adjunto; Diretor do Departamento de Ortopedia e Traumatologia da Santa Casa de Misericórdia de São Paulo - SC MSP - São Paulo (SP), Brasil.
} 
rença estatística no comportamento das curvas durante o primeiro ano de seguimento nas curvas torácica, lombar ou na cifose. Quando analisadas de forma quantitativa, não se encontrou diferença estatística nas curvas torácica $(\mathrm{p}=0,052)$ e lombar $(\mathrm{p}=0,332)$ no período pré-operatório entre os três grupos. Os três instrumentais apresentaram comportamento semelhante, não havendo diferença quantitativa na correção das curvas torácica $(\mathrm{p}=0,052)$ e lombar $(p=0,267)$ nos períodos pósoperatório imediato e após um ano de seguimento. Conclusões: as três estratégias de instrumentação rendem resultados radiográficos similares em pacientes portadores de EIA flexíveis. of the curves during the first year of follow-up concerning the thoracic and lumbar curves or the kyphosis. In a quantitative analysis, no statistical difference was found in the thoracic curves $(p=0.052)$ or lumbar curves $(p=0.332)$ in the preoperative period among the three groups. The three instruments showed similar behaviour patterns, with no quantitative differences in the correction of thoracic curves $(p=0.052)$ and lumbar curves $(p=0.267)$ during the immediate postoperative period and after one year of follow-up. Conclusions: the three instrumentations yield similar radiographic findings in patients with AIS. tamiento durante el primer año de seguimiento de las curvas torácica o lumbar y de la cifosis. Cuando se analizan cuantitativamente, no se encontraron diferencias estadisticas en las curvas torácica $(p=0,052)$ o lumbar $(p=0,332)$ en el período preoperatorio entre los tres grupos. Los tres instrumentos mostraron patrones similares, sin diferencias cuantitativas en la corrección de las curvas torácica $(p=0,052)$ y lumbar $(p=0,267)$ durante el periodo postoperatorio inmediato y después del uno año de seguimiento. Conclusiones: las tres estrategias de instrumentación han obtenido hallazgos radiográficos similares en pacientes con EIA flexible.
DESCRITORES: Escoliose; Escoliose/cirurgia; Escoliose/ terapia; Doenças da coluna vertebral/cirurgia; Fusão vertebral/instrumentação
KEYWORDS: Scoliosis; Scoliosis/ surgery; Scoliosis/therapy; Spinal diseases/surgery; Spinal fusion/instrumentation
DESCRIPTORES: Escoliosis; Escoliosis/cirugía; Escoliosis/ terapia; Enfermedades de la columna vertebral/ cirugía; Fusión vertebral/ instrumentación

\section{INTRODUÇÃO}

A escoliose idiopática do adolescente (EIA) pode ser definida como uma deformidade tridimensional com rotação dos corpos vertebrais e inclinação no plano coronal que excede $10^{\circ}$ pelo método de medição de Cobb numa radiografia ortostática em indivíduos entre 10 e 18 anos de ida$\mathrm{de}^{1}$. Sua etiologia permanece desconhecida e sua prevalência é de, aproximadamente, 2 a 3\% para as deformidades de menor valor angular, chegando entre 0,1 a $0,3 \%$ para as curvaturas acima de $30^{\circ}$. Não há predileção por sexo nas deformidades de menor valor angular, mas há predileção pelo sexo feminino nas curvas mais graves ${ }^{1}$.

$\mathrm{Na}$ literatura, menos de $10 \%$ dessas deformidades não necessitam de tratamento por não alcançar valores angulares acima de $20^{\circ}$. As deformidades entre $20^{\circ}$ e $40^{\circ}$ são tratadas por meio de órteses aplicadas em tempo integral e as curvaturas acima de $40^{\circ}$ são tratadas cirurgicamente. As deformidades negligenciadas, principalmente nos pacientes que apresentam progressão do valor angular, são as responsáveis pelo aumento da taxa de mortalidade e repercussões negativas como dores residuais na coluna vertebral e alterações psicossociais ${ }^{1}$. Os objetivos do tratamento são: estabilizar a progressão da deformidade, manter o tronco balanceado e compensado nos planos coronal e sagital ${ }^{2,3}$. $\mathrm{O}$ tratamento cirúrgico alcança os objetivos por meio do sucesso de uma artrodese sólida e correção do valor angular da deformidade ${ }^{4}$.
Um fator de destaque na história natural da escoliose idiopática do adolescente é a progressão da curvatura, a qual está relacionada com o potencial de crescimento do indivíduo, idade da menarca no sexo feminino, ossificação da apófise do osso ilíaco - evidenciado pelos critérios propostos por Risser $^{5}$ - e a maturação sexual - estimada pela classificação de Tanner ${ }^{6}$. Além disso, o padrão e a magnitude da deformidade relacionam-se com a progressão, sendo as duplas curvas e as de maior valor angular aquelas de maior risco ${ }^{1}$.

Nos pacientes com deformidades acima de $40^{\circ}$ e com os fatores de progressão acima citados, há indicação do tratamento cirúrgico, que envolve correção e artrodese das curvas. Muitos autores postulam que quanto menor o número de segmentos submetidos a artrodese, menores são as complicações, como perda sanguínea e risco de pseudoartrose $^{2}$. Os implantes metálicos empregados no tratamento cirúrgico da escoliose idiopática do adolescente estão evoluindo desde 1960, quando houve a introdução do instrumental de Harrington ${ }^{3}$ que permitia uma correção limitada e artrodeses mais longas e, como inconveinente, o paciente poderia apresentar perda dos contornos fisológicos da coluna caracterizando o flat back esteticamente idesejável ${ }^{7}$.

Em 1984, com a introdução do instrumental de CotrelDubousset, houve possibilidade de correção tridimensional de forma consistente e reprodutível em outros trabalhos ${ }^{4,8}$. 
Inicialmente, os implantes eram compostos apenas com ganchos e, com a evolução do material cirúrgico, os parafusos pediculares passaram a ser disponíveis para correção das deformidades. Esses implantes denominados de terceira geração permitiram uma instrumentação segmentar da coluna vertebral contribuindo para a correção da deformidade, diminuindo o número de vértebras envolvidas na artrodese, mas os efeitos na correção da rotação vertebral permaneciam questionáveis utilizando-se ganchos na coluna torácica ${ }^{4,8-10}$.

A aplicação de montagens apenas com parafusos pediculares passou a ser estudada a partir de 1995. As vantagens dos parafusos torácicos pediculares, quando comparado com os ganchos, incluem maior correção tridimensional e coronal $^{3,11,12-17}$. Entretanto, a maior curva de aprendizado ${ }^{7}$, bem como a morfologia alterada da coluna vertebral, dificultam a colocação dos parafusos, aumentando os riscos de eventuais lesões neurológicas iatrogênicas ${ }^{3,16,17}$.

O objetivo deste estudo foi comparar o desfecho radiográfico dos portadores de EIA submetidos a tratamento cirúrgico empregando instrumentação com ganchos, híbrida e parafusos pediculares.

\section{MÉTODOS}

Após aprovação pelo Conselho de Ética em Pesquisa (CEP), foram avaliados retrospectivamente radiografias de 49 pacientes portadores de EIA submetidos a tratamento cirúrgico através de via de acesso posterior pelo grupo da coluna do Departamento de Ortopedia e Traumatologia da Faculdade de Ciências Médicas da Santa Casa de São Paulo, de junho de 1997 a novembro de 2008. Observaram- se 45 pacientes do sexo feminino $(91,8 \%)$ e 4 do sexo masculino $(8,2 \%)$, com idades variando de 11 a 25 anos (média de 15,2 anos) na época do tratamento cirúrgico.

Foram incluídos no estudo todos os pacientes portadores de EIA tratada cirurgicamente mediante uso de ganchos, montagens híbridas ou apenas parafusos pediculares, com documentação radiográfica composta de exames pré-operatórios nas incidências de frente e perfil em ortostase e inclinações laterais em posição supina, e exames pós-operatórios imediato, com um ano de seguimento nas incidências de frente e perfil em ortostase. Foram excluídos os pacientes portadores de escolioses secundárias a outras etiologias, pacientes sem documentação radiográfica satisfatória e aqueles com seguimento menor de um ano.

Os pacientes foram subdivididos em três grupos, de acordo com o tipo de instrumentação utilizada na cirurgia, sendo alocados no grupo um os pacientes submetidos a artrodese com ganchos na coluna torácica e lombar, no grupo dois os pacientes submetidos a artrodese com instrumentação híbrida (parafusos pediculares no coluna lombar e ganchos na torácica) e no grupo três aqueles submetidos a artrodese com instrumentação apenas com parafusos pediculares. Todos os pacientes foram classificados segundo King $^{18}$ e Lenke ${ }^{19}$ (Tabela 1).
A comparação das correções das curvas torácica principal e compensatória, entre os três grupos de acordo com o período de avaliação foi realizada por meio de análise de variância com métodos repetidos e comparações múltiplas (comparações pareadas). O nível de significância adotado foi de 5\% sendo utilizado o software SPSS (v.13) para as análises estatísticas.

\section{RESULTADOS}

Dos 49 pacientes avaliados, 13 (26,5\%) foram submetidos à correção e artrodese apenas com ganchos, em 25 (51\%) utilizou-se instrumentação híbrida e em $11(22,4 \%)$, utilizaram-se apenas parafusos pediculares.

A distribuição das curvas segundo a classificação de King e Lenke mostrou predominância das curvas do tipo King III (53\%) e Lenke I (44,9\%), porém foi muito heterogênea, não permitindo uma análise estatística com significância menor ou igual a 5\%. Dois pacientes (indivíduos 18 e 37) não foram classificados segundo King por apresentarem curvas lombares (Tabela 1). As medidas da cifose não mostraram diferença estatística entre os três grupos em nenhum dos períodos estudados ( $\mathrm{p}>0,999)$.

Quando analisadas de forma quantitativa, não se encontrou diferença estatística nas curvas torácicas $(p=0,052)$ e lombares $(\mathrm{p}=0,332)$ no período pré-operatório entre os três grupos, sendo portanto, homogêneos (Tabela 2).

Ao longo do tempo, os três instrumentais apresentaram comportamento semelhante, não havendo diferença quantitativa na correção das curvas torácica $(\mathrm{p}=0,052)$ (Figura 1) e lombar ( $p=0,267)$ (Figura 2) nos períodos pós-operatório imediato e após um ano de seguimento.

\section{DISCUSSÃO}

Apesar do uso mais frequente dos parafusos pediculares, a aplicação dos ganchos, em especial na coluna torácica, permanece opção de fixação das hastes metálicas com menor risco de invasão no canal vertebral ou na lesão vascular?. Nenhum trabalho apresentou resultados consistentes a favor do uso dos parafusos pediculares e, em alguns estudos, a utilização de ganchos na coluna torácica mostrou-se mais eficaz na correção do balanço sagital comparado com uso de parafusos pediculares $^{16,17}$. Da mesma forma, em nosso estudo verificamos que a opção pelo uso somente de ganchos não refletiu prejuízo ao fim do seguimento de um ano em nossos pacientes.

Neste estudo não se observou diferença ao longo do tempo no comportamento dos três instrumentais nas correções das curvas torácica e lombar. A perda da correção foi um fator homogêneo que não se relacionou diretamente com a escolha do implante empregado. Na literatura, a perda de correção ao longo do seguimento pós-operatório é relatada, mas não encontramos descrição específica que relacionasse diretamente maiores perdas na correção do valor angular ao tipo de implante empregado.

Alguns fatores devem ser levados em conta para justificarmos a falta de correlação estatística evidente em nosso estudo. A amostra analisada constitui um grupo bastante 
TABELA 1 - Distribuição dos pacientes conforme instrumentação utilizada e classificação segundo King $^{18}$ e Lenke ${ }^{19}$

\begin{tabular}{|c|c|c|c|c|c|c|c|c|}
\hline $\mathrm{n}$ & Sexo & $\begin{array}{c}\text { Instrumental } \\
\text { utilizado }\end{array}$ & $\begin{array}{c}\text { Torácica } \\
\text { proximal } \\
\text { (graus) }\end{array}$ & $\begin{array}{c}\text { Torácica } \\
\text { principal } \\
\text { (graus) }\end{array}$ & $\begin{array}{l}\text { Lombar } \\
\text { (graus) }\end{array}$ & $\begin{array}{l}\text { Cifose } \\
\text { (graus) }\end{array}$ & $\begin{array}{c}\text { Classificação } \\
\text { King }\end{array}$ & $\begin{array}{c}\text { Classificação } \\
\text { Lenke }\end{array}$ \\
\hline 1 & $\mathrm{~F}$ & Ganchos & 35 & 51 & 20 & 22 & III & IIAN \\
\hline 2 & $\mathrm{~F}$ & Ganchos & 40 & 41 & 26 & 15 & III & IIBN \\
\hline 3 & $\mathrm{~F}$ & Híbrido & 34 & 56 & 36 & 26 & III & IBN \\
\hline 4 & $\mathrm{~F}$ & Híbrido & 45 & 100 & 60 & 71 & III & IVB+ \\
\hline 5 & $\mathrm{~F}$ & Ganchos & 13 & 51 & 30 & 19 & III & $\mathrm{IBN}$ \\
\hline 6 & $\mathrm{~F}$ & Ganchos & 16 & 50 & 42 & 24 & III & $\mathrm{IBN}$ \\
\hline 7 & M & Híbrido & 15 & 55 & 30 & 54 & III & $\mathrm{IC}+$ \\
\hline 8 & $\mathrm{~F}$ & Híbrido & 10 & 58 & 24 & 30 & IV & IAN \\
\hline 9 & $\mathrm{~F}$ & Parafusos & 0 & 45 & 74 & 9 & I & VIC- \\
\hline 10 & M & Parafusos & 70 & 88 & 45 & 49 & III & IVB + \\
\hline 11 & $\mathrm{~F}$ & Híbrido & 0 & 100 & 70 & 55 & ॥ & $\mathrm{IIIC}+$ \\
\hline 12 & $\mathrm{~F}$ & Parafusos & 10 & 56 & 30 & 4 & III & IAN \\
\hline 13 & $\mathrm{~F}$ & Híbrido & 60 & 120 & 60 & 55 & III & $\mathrm{IIB}+$ \\
\hline 14 & $\mathrm{~F}$ & Híbrido & 62 & 70 & 45 & 50 & III & $\| C+$ \\
\hline 15 & $\mathrm{~F}$ & Parafusos & 20 & 58 & 65 & 13 & I & IIICN \\
\hline 16 & $\mathrm{~F}$ & Híbrido & 46 & 68 & 50 & 40 & $\|$ & $\| C N$ \\
\hline 17 & $\mathrm{~F}$ & Híbrido & 40 & 60 & 34 & 20 & II & $I I C N$ \\
\hline 18 & $\mathrm{~F}$ & Híbrido & 0 & 40 & 87 & 30 & $N C^{*}$ & $\mathrm{VICN}$ \\
\hline 19 & $\mathrm{~F}$ & Híbrido & 25 & 77 & 66 & 9 & II & IC- \\
\hline 20 & $\mathrm{~F}$ & Híbrido & 40 & 128 & 66 & 90 & III & IVB+ \\
\hline 21 & $\mathrm{~F}$ & Parafusos & 20 & 42 & 56 & 22 & I & VCN \\
\hline 22 & $\mathrm{~F}$ & Híbrido & 17 & 60 & 20 & 42 & III & IAN \\
\hline 23 & $\mathrm{~F}$ & Parafusos & 35 & 75 & 55 & 40 & ॥ & IICN \\
\hline 24 & M & Híbrido & 10 & 68 & 40 & 50 & III & $\mathrm{A}+$ \\
\hline 25 & $\mathrm{~F}$ & Híbrido & 30 & 65 & 53 & 34 & II & IBN \\
\hline 26 & $\mathrm{~F}$ & Híbrido & 0 & 54 & 54 & 14 & II & IBN \\
\hline 27 & $\mathrm{~F}$ & Parafusos & 30 & 70 & 45 & 20 & III & $\mathrm{IBN}$ \\
\hline 28 & $\mathrm{~F}$ & Parafusos & 20 & 52 & 22 & 16 & III & IAN \\
\hline 29 & $\mathrm{~F}$ & Híbrido & 10 & 67 & 68 & 32 & $\|$ & $\mathrm{ICN}$ \\
\hline 30 & $\mathrm{~F}$ & Híbrido & 50 & 64 & 20 & 12 & IV & IIAN \\
\hline 31 & $\mathrm{~F}$ & Híbrido & 0 & 46 & 42 & 10 & ॥ & IIBN \\
\hline 32 & $\mathrm{~F}$ & Parafusos & 25 & 60 & 45 & 12 & III & $\mathrm{IBN}$ \\
\hline 33 & $\mathrm{~F}$ & Híbrido & 30 & 55 & 32 & 22 & III & IIAN \\
\hline 34 & $\mathrm{~F}$ & Híbrido & 5 & 55 & 63 & 19 & I & VCN \\
\hline 35 & $\mathrm{~F}$ & Ganchos & 37 & 60 & 40 & 40 & III & IIBN \\
\hline 36 & $\mathrm{~F}$ & Ganchos & 15 & 50 & 38 & 12 & II & IBN \\
\hline 37 & $\mathrm{~F}$ & Híbrido & 0 & 55 & 110 & 70 & $\mathrm{NC}^{*}$ & $\mathrm{VIC}+$ \\
\hline 38 & $\mathrm{~F}$ & Híbrido & 17 & 48 & 48 & 52 & $\|$ & $\mathrm{IC}+$ \\
\hline 39 & $\mathrm{~F}$ & Híbrido & 20 & 80 & 42 & 35 & III & IAN \\
\hline 40 & $\mathrm{~F}$ & Ganchos & 30 & 55 & 30 & 28 & III & $\| A N$ \\
\hline 41 & $\mathrm{~F}$ & Parafusos & 20 & 50 & 21 & 10 & IV & IAN \\
\hline 42 & $\mathrm{~F}$ & Ganchos & 25 & 76 & 72 & 19 & ॥ & IIICN \\
\hline 43 & $\mathrm{~F}$ & Ganchos & 24 & 60 & 80 & 29 & ॥ & IIICN \\
\hline 44 & $\mathrm{~F}$ & Híbrido & 40 & 100 & 60 & 60 & III & IVB+ \\
\hline 45 & $\mathrm{~F}$ & Ganchos & 35 & 68 & 39 & 13 & II & $\| C N$ \\
\hline 46 & $\mathrm{~F}$ & Ganchos & 25 & 50 & 36 & 40 & III & IAN \\
\hline 47 & $\mathrm{~F}$ & Ganchos & 23 & 48 & 27 & 35 & III & IAN \\
\hline 48 & $\mathrm{~F}$ & Ganchos & 15 & 49 & 30 & 16 & III & IAN \\
\hline 49 & M & Parafusos & 20 & 59 & 37 & 20 & III & IB- \\
\hline
\end{tabular}

NC*: pacientes sem classificação possível segundo King e/ou Lenke. 
TABELA 2 - Distribuição das curvas torácica e lombar segundo instrumentação utilizada e correção nos períodos pós-operatório imediato e após um ano de seguimento

\begin{tabular}{llcccccc}
\hline & \multicolumn{2}{c}{$\begin{array}{c}\text { Pré-operatório } \\
\text { (graus) }\end{array}$} & $\begin{array}{c}\text { Pós-operatório imediato } \\
\text { (graus) }\end{array}$ & \multicolumn{2}{c}{$\begin{array}{c}\text { Pós-operatório 1 ano } \\
\text { (graus) }\end{array}$} \\
\cline { 3 - 8 } & $\begin{array}{c}\text { Curva } \\
\text { torácica }\end{array}$ & $\begin{array}{c}\text { Curva } \\
\text { lombar }\end{array}$ & $\begin{array}{c}\text { Curva } \\
\text { torácica }\end{array}$ & $\begin{array}{c}\text { Curva } \\
\text { lombar }\end{array}$ & $\begin{array}{c}\text { Curva } \\
\text { torácica }\end{array}$ & $\begin{array}{c}\text { Curva } \\
\text { lombar }\end{array}$ \\
\hline Ganchos & Mínimo & 41 & 20 & 4 & 1 & 12 & 7 \\
& Máximo & 76 & 80 & 52 & 42 & 52 & 50 \\
& Média & 54,5 & 39,2 & 23,6 & 18,9 & 26,3 & 23 \\
& Desvio padrão & 9,3 & 17,5 & 11,2 & 11,4 & 10,3 & 14 \\
Híbrido & Mínimo & 40 & 20 & 18 & 0 & 20 & 0 \\
& Máximo & 128 & 110 & 100 & 50 & 105 & 60 \\
& Média & 69,9 & 51,2 & 36,2 & 22,6 & 39 & 26,6 \\
& Desvio padrão & 22,7 & 20,9 & 18,7 & 13,7 & 18,3 & 14,1 \\
Parafusos & Mínimo & 42 & 21 & 3 & 0 & 5 & 0 \\
& Máximo & 88 & 74 & 85 & 39 & 85 & 42 \\
& Média & 59,5 & 45 & 25,7 & 17 & 29,1 & 22,3 \\
& Desvio padrão & 13,5 & 16,9 & 21,7 & 13,2 & 23 & 13,1 \\
\hline
\end{tabular}

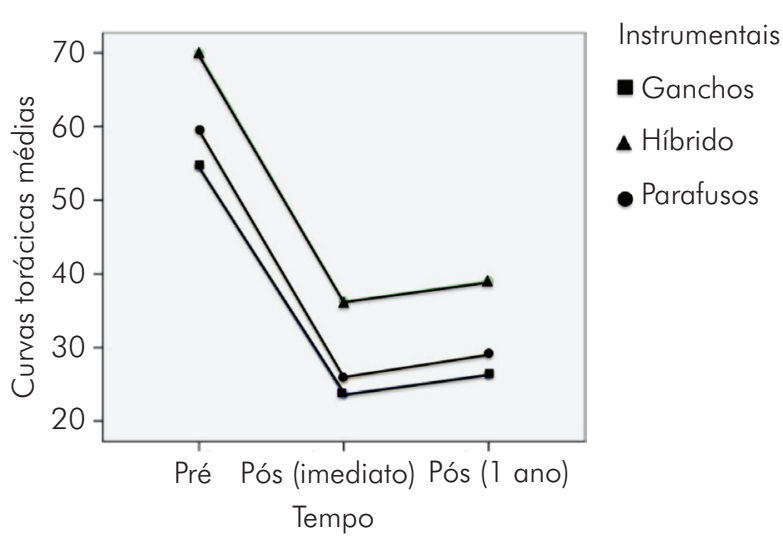

Figura 1

Comportamento das curvas torácicas principais ao longo do tempo utilizando os diferentes instrumentais.

heterogêneo, principalmente quando classificamos as curvas segundo os critérios descritos por Lenke et al. ${ }^{19}$ já que essa classificação compreende um grande número de variáveis e subtipos.

Outro ponto relevante diz respeito à análise da correção das deformidades, a qual foi realizada de forma qualitativa, utilizando-se modelos matemáticos, pois a amostra dentre os três grupos era heterogênea, com muitos indivíduos no grupo tratados com instrumentação híbrida em detrimento dos demais grupos, mas com uma análise estatística com significância de 5\%.

Deve-se ainda levar em consideração a curva de aprendizado na utilização dos parafusos pediculares na coluna torácica ${ }^{7}$, a preferência do cirurgião em relação ao instrumental, a manobra de correção da deformidade ${ }^{8} \mathrm{e}$ a estratégia de colocação dos parafusos pediculares. Em nossa casuística, consideramos esse fator um item relevante frente à análise dos resultados.

Não houve padronização na utilização dos parafusos pediculares na coluna torácica, diferentemente de

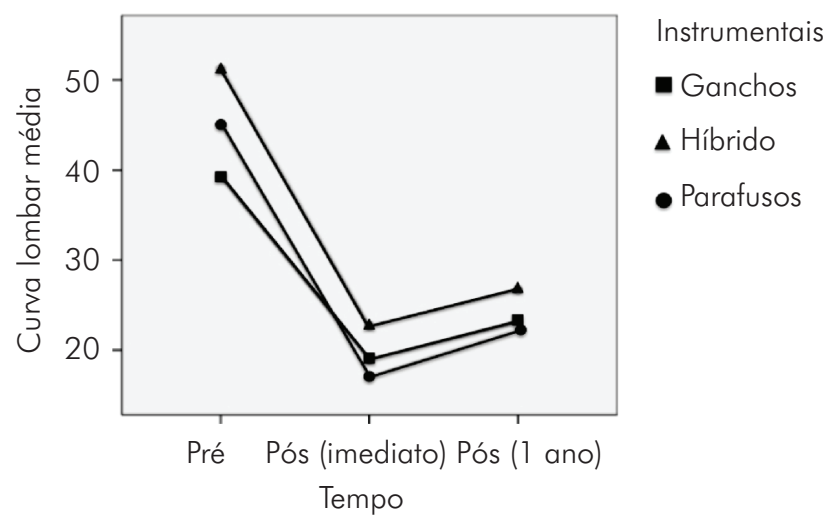

Figura 2

Comportamento das curvas lombares ao longo do tempo utilizando os diferentes instrumentais.

outros estudos que alocavam os parafusos nas vértebras estratégicas conforme preconizado por CotrelDubousset, fato que talvez tenha alguma interferência na correção final. A justificativa para esse fato é dada, pois uma variedade muito grande de curvas foi incluída neste estudo e, assim, houve necessidade de diferentes estratégias de instrumentação que variaram de acordo com o valor angular, a rigidez das curvas escolióticas e a estratégia de correção.

\section{CONCLUSÃO}

Em relação ao desfecho radiográfico este estudo não mostrou diferença estatística na correção das curvas torácica principal e lombar com a utilização de ganchos, montagem híbrida ou apenas parafusos pediculares. Faltam na literatura estudos prospectivos randomizados, comparando os instrumentais para que haja uma melhor avaliação de custo versus benefício na utilização desses diferentes instrumentais no tratamento da EIA. 


\section{REFERÊNCIAS}

1. Lonstein JE. Scoliosis: surgical versus nonsurgical treatment. Clin Orthop Relat Res. 2006;(443):248-59.

2. Cheh G, Lenke LG, Lehman RA Jr, Kim YJ, Nunley R, Bridwell KH. The reliability of preoperative supine radiographs to predict the amount of curve flexibility in adolescent idiopathic scoliosis. Spine (Phila Pa 1976). 2007;32(24):2668-72.

3. Aubin CE, Labelle H, Ciolofan OC. Variability of spinal instrumentation configurations in adolescent idiopathic scoliosis. Eur Spine J. 2007;16(1):57-64.

4. Helenius I, Remes V, Yrjönen T, Ylikoski M, Schlenzka D, Helenius M, et al. Harrington and Cotrel-Dubousset instrumentation in adolescent idiopathic scoliosis. Long-term functional and radiographic outcomes. J Bone Joint Surg Am. 2003;85A(12):2303-9.

5. Risser JC. The iliac apophysis: an invaluable sign in the management of scoliosis. Clin Orthop. 1958;(11):111-9.

6. Tanner JM. Some main features of normal growth in children. In: Zorab PA (Ed.). Scoliosis and Growth. Edinburgh, UK: Churchill Livingstone; 1971. p. 14-28.

7. Kuklo TR, Potter BK, Lenke LG, Polly DW Jr, Sides B, Bridwell KH. Surgical revision rates of hooks versus hybrid versus screw versus combined anteroposterior spinal fusion for adolescent idiopathic scoliosis. Spine (Phila Pa 1976). 2007;32(20):2258-64.

8. Delorme S, Labelle H, Aubin CE, de Guise JA, Rivard CH, Poitras $\mathrm{B}$, et al. Intraoperative comparison of two instrumentation techniques for the correction of adolescent idiopathic scoliosis. Rod rotation and translation. Spine (Phila Pa 1976). 1999;24(19):2011-7.
9. Wright JG, Donaldson S, Howard A, Stephens D, Alman B, Hedden D. Are surgeons' preferences for instrumentation related to patient outcomes? A randomized clinical trial of two implants for idiopathic scoliosis. J Bone Joint Surg Am. 2007;89(12):2684-93.

10.Muschik M, Schlenzka D, Robinson PN, Kupferschmidt C. Dorsal instrumentation for idiopathic adolescent thoracic scoliosis: rod rotation versus translation. Eur Spine J. 1999;8(2):93-9.

11.Dobbs MB, Lenke LG, Kim YJ, Kamath G, Peelle MW, Bridwell KH. Selective posterior thoracic fusions for adolescent idiopathic scoliosis: comparison of hooks versus pedicle screws. Spine (Phila Pa 1976). 2006;31(20):2400-4.

12. Watanabe K, Lenke LG, Bridwell KH, Kim YJ, Watanabe K, et al. Comparison of radiographic outcomes for the treatment of scoliotic curves greater than 100 degrees: wires versus hooks versus screws. Spine (Phila Pa 1976). 2008;33(10):1084-92.

13.Hee HT, Yu ZR, Wong HK. Comparison of segmental pedicle screw instrumentation versus anterior instrumentation in adolescent idiopathic thoracolumbar and lumbar scoliosis. Spine (Phila Pa 1976). 2007;32(14):1533-42.

14. Senaran H, Shah SA, Gabos PG, Littleton AG, Neiss G, Guille JT. Difficult thoracic pedicle screw placement in adolescent idiopathic scoliosis. J Spinal Disord Tech. 2008;21(3):187-91
15.Lonner BS, Auerbach JD, BoachieAdjei O, Shah SA, Hosogane N, Newton PO. Treatment of thoracic scoliosis: are monoaxial thoracic pedicle screws the best form of fixation for correction? Spine (Phila $\mathrm{Pa}$ 1976). 2009;34(8):845-51.

16.Liljenqvist U, Lepsien U, Hackenberg L, Niemeyer T, Halm H. Comparative analysis of pedicle screw and hook instrumentation in posterior correction and fusion of idiopathic thoracic scoliosis. Eur Spine J. 2002;11(4):336-43.

17.Kim YJ, Lenke LG, Kim J, Bridwell $\mathrm{KH}$, Cho SK, Cheh G, et al. Comparative analysis of pedicle screw versus hybrid instrumentation in posterior spinal fusion of adolescent idiopathic scoliosis. Spine (Phila $\mathrm{Pa}$ 1976). 2006;31(3):291-8.

18.King HA, Moe JH, Bradford DS, Winter RB. The selection of fusion levels in thoracic idiopathic scoliosis. J Bone Joint Surg Am. 1983;65(9):1302-13.

19.Lenke LG, Betz RR, Harms J, Bridwell KH, Clements DH, Lowe TG, et al. Adolescent idiopathic scoliosis: a new classification to determine extent of spinal arthrodesis. J Bone Joint Surg Am. 2001;83(8):1169-81.

\section{Correspondência}

Robert Meves

Rua Dr. Cesário Motta Jr, 112 - Vila Buarque

CEP: 01221 - 020 - São Paulo (SP), Brasil

Tel.: (1 1 ) 3222-6866

E-mail:robertmeves@hotmail.com 